\title{
Rezension: David Henseler, Datenschutz bei drohnengestützter Datenbearbeitung durch Private
}

Der Einsatz von Drohnen durch Private nimmt stetig zu und wirft gewichtige datenschutzrechtliche Fragen auf: Wer eine Drohne hört oder sieht, weiss meistens nicht, ob er oder sie gerade beobachtet wird, ob dabei aufgezeichnet wird und wer für die Drohne verantwortlich ist. Eine neu erschienene Dissertation untersucht, wie das Recht auf informationelle Selbstbestimmung bei der drohnengestützten Datenbearbeitung durch Private durchgesetzt werden kann. Die Studie analysiert sowohl das neue Datenschutzgesetz als auch die Vorschriften zur Drohnenfliegerei und präsentiert gestützt darauf konkrete Vorschläge für eine Gesetzesanpassung.

\section{Einleitung}

II. Inhalt

Zitiervorschlag:

NULA FREI, Rezension: David Henseler, Datenschutz bei drohnengestützter Datenbearbeitung durch Private, sui generis 2022, S. 1

Nula Frei, Dr. iur., Oberassistentin am Institut für Europarecht der Universität Freiburg (nula.frei@unifr.ch).

URL: sui-generis.ch/198 


\section{Einleitung}

1 Ein lieblicher Sonntagnachmittag im Spätfrühling, wir sitzen zu dritt bei Kaffee und Kuchen im Garten, als ein Surren in der Luft vernehmbar wird. Mal etwas näher, dann wieder weiter weg. Und dann wird sie sichtbar, die Drohne. Sie fliegt heran und kommt in etwa fünf Metern Höhe direkt über unserem Kaffeetisch zum Stehen mit ihrem unangenehmen, bienenschwarmähnlichen Summton. Wir gestikulieren, machen verscheuchende Bewegungen, aber das Ding schwebt weiter über uns, nähert sich sogar noch, als würde es uns verhöhnen. Erst als wir anfangen, Steine vom Boden aufzuheben und Wurfbewegungen anzudeuten, zieht es davon und verschwindet hinter einem der Nachbarshäuser.

2 Ich kann es nicht leugnen: Spätestens seit dieser Begegnung hege ich eine geradezu unversöhnliche Abneigung gegen privat genutzte Drohnen und auch gewisse Abschussfantasien sind mir nicht fremd. Dennoch (oder vielleicht gerade deswegen) habe ich das Buch «Datenschutz bei drohnengestützter Datenbearbeitung durch Private» von David Henseler, das auf seiner 2020 an der Universität Zürich angenommenen Dissertation beruht, mit Interesse gelesen. Die Studie hat zum Ziel, zu untersuchen, wie das Recht auf informationelle Selbstbestimmung bei der drohnengestützten Datenbearbeitung durch Private durchgesetzt werden kann.

\section{Inhalt}

3 Der Autor analysiert sowohl das Datenschutzgesetz als auch die luftfahrtrechtlichen Vorschriften zur Drohnenfliegerei und entwirft gestützt darauf einen konkreten Gesetzgebungsvorschlag. Seine Studie steht, so führt er einleitend aus, vor dem Hintergrund einer stetig zunehmenden Nutzung von Drohnen durch Privatpersonen (sog. «Hobbydrohnen») bei gleichzeitig bestehender Skepsis in der Bevölkerung (S. 4 ff.). Diese Skepsis rühre hauptsächlich von den mannigfaltigen Möglichkeiten zur Bearbeitung personenbezogener Daten durch die heute handelsüblichen Drohnen, welche mit Foto- oder Videokameras, Infrarot- oder Wärmesensoren und teilweise auch mit Mikrofonen ausgestattet sind (S. 43). Problematisch ist dabei, so Henseler, dass in der Regel nicht erkennbar ist, ob eine Drohne gerade fotografiert oder filmt, ob das Aufgenommene live übertragen oder aufgezeichnet wird. Ebenso bleibe regelmässig unklar, wer für die Drohne verantwortlich ist. Kurioserweise gibt es zum Einsatz von Drohnen kaum Literatur und Rechtsprechung, wie der Autor in seiner Einführung darlegt, und so ist die Studie von Henseler zweifelsfrei von besonderer Aktualität und Relevanz.
Mit gerade mal 177 eher gross bedruckten Seiten ist das 4 Buch für eine Dissertation ziemlich kurz, dadurch aber auch besonders angenehm zu lesen. Von überflüssigem Sprachgeplänkel oder redundanten Ausführungen gänzlich unbelastet, fokussiert sich die Studie in fünf Teilen, eingerahmt von einer Einleitung und einem Schlusswort, auf das Wesentliche.

Nach der Einleitung, in welcher Forschungsfrage, Rele- 5 vanz des Themas und Stand der Wissenschaft dargestellt werden, führt der Autor in Teil 1 in die rechtlichen, begrifflichen und technischen Grundlagen der Drohnenfliegerei ein. Dass der Autor seine Analyse auf den Entwurf des Datenschutzgesetzes in der vom Nationalrat am 25. September 2019 beschlossenen Version stützt, während die finale Version von 20. September 2020 datiert und dazwischen noch Beratungen von Stände- und Nationalrat (in der Wintersession 2019 und Frühjahrssession 2020) lagen, ist etwas bedauerlich und schränkt die Qualität der Arbeit zumindest der Form nach ein, zumal der Autor diese Einschränkung nicht näher rechtfertigt. Es ist ihm aber zugute zu halten, dass im Juli 2020, als das Buch publiziert wurde, noch nicht absehbar war, ob das Gesetz in der darauffolgenden Herbstsession verabschiedet würde. Immerhin sind - soweit ersichtlich - die nach dem Beschluss des Nationalrats im September 2019 erfolgten Änderungen an der Vorlage nicht derart weitgehend, dass die Ergebnisse der Studie grundlegend in Frage gestellt würden (einen solchen Vergleich durch den Autor sucht man aber im Buch vergeblich). Auch die zweite wichtige Rechtsquelle, auf die sich die Studie stützt, nämlich die EU-Drohnenverordnungen, die von der Schweiz gestützt auf das bilaterale Luftverkehrsabkommen zu übernehmen sind, ist in der Schweiz noch nicht vollständig umgesetzt. Da es hier aber zu Verzögerungen im Gesetzgebungsprozess kam (und noch immer kommt), und eines der wesentlichen Elemente von Henselers Studie die Formulierung eines Umsetzungsvorschlags ist, wiegt dies vorliegend weniger schwer.

In Teil 2 widmet sich der Autor den grundrechtlichen 6 Aspekten der drohnengestützten Datenbearbeitung. Ein Schwerpunkt liegt dabei auf der Schutzpflichtendogmatik, die für die Studie von Bedeutung ist, weil der Autor daraus am Ende der Studie seine Forderung nach einem verstärkten Eingreifen des Gesetzgebers ableiten wird. Anschliessend prüft er mögliche, durch die drohnengestützte Datenbearbeitung tangierte Grundrechte, darunter das Recht auf Privatsphäre, namentlich die Achtung der Wohnung (wozu auch Balkone oder Gärten gehören), und die Achtung des Privatlebens, wobei der Autor hier zutreffenderweise auch die Problematik eines möglichen «chilling effects» hervorhebt, der durch das Gefühl des Beobachtetwerdens entsteht, insbesondere wenn Drohnen aus voyeuristischen Beweggründen oder aus blosser 
Neugierde zur Beobachtung eingesetzt werden (die über dem sonntagnachmittäglichen Kaffeetisch kreisende Drohne lässt grüssen). Im Zentrum steht richtigerweise aber, so der Autor, das Recht auf informationelle Selbstbestimmung, das sich nach h.L. aus Art. 13 Abs. 2 BV1 ergibt, denn dort stellen sich die meisten und relevantesten Rechtsprobleme. Jede unerwünschte Datenbearbeitung mittels Drohnen, also auch das Aufnehmen von menschlichen Foto- oder Videobildern, stellt eine Einschränkung dieses Grundrechts dar und bewirkt, wenn die Einschränkung von Privaten ausgeht, eine staatliche Schutzpflicht.

7 Sodann nimmt der Autor in den folgenden zwei Teilen eine Bestandesaufnahme des Datenschutzrechts (Teil3) und des Luftfahrtrechts (Teil4) vor. Ziel ist zu überprüfen, «ob der Gesetzgeber seinen grundrechtlichen Schutzpflichten im Kontext der informationellen Selbstbestimmung nachgekommen ist» (S. 73). In Bezug auf das Datenschutzrecht kommt der Autor zum Schluss, dass die Drohnenfliegerei mit verschiedenen rechtlichen Vorgaben in Konflikt kommt. Bereits in Teil 1 hatte der Autor festgestellt, dass bei der drohnengestützten Datenbearbeitung regelmässig auch besonders schützenswerte Personendaten generiert werden - dann nämlich, wenn durch die erstellten Foto- oder Videoaufnahmen, die heutzutage auch von weit her hochauflösendes Bildmaterial produzieren (so können z.B. Autokennzeichen aus zwei Kilometern Entfernung erkannt werden, S. 43) oder Informationen wie die Rassenzugehörigkeit oder die religiöse Ansicht einer Person erkennbar werden (S.34). Zu den datenschutzrechtlichen Grundsätzen, die bei der Drohnenfliegerei verletzt sind, gehört das Transparenzgebot: Die betroffenen Personen sind sich normalerweise über den Zweck sowie die Art und den Umfang der drohnengestützt bearbeiteten Daten nicht im Klaren und können dies auch nicht erkennen (S. 86). Damit ist auch davon auszugehen, dass bei der drohnengestützten Datenbearbeitung keine rechtskonforme Information der betroffenen Personen erfolgt, sei es, weil sich Drohnenbetreiber einer entsprechenden Pflicht nicht bewusst sind oder aber diese nicht erfüllen wollen (S. 98). Da Drohnenbetreiber sich in der Regel auch nicht zu erkennen geben, können Betroffene die ihnen zustehenden Rechte, namentlich das Auskunftsrecht, nicht wahrnehmen(S.102). Das Auskunftsrecht kann in diesem Kontext die ihm zugedachte Funktion nicht erfüllen (S. 105). Dadurch ist aber auch keine rechtsgültige Einwilligung der Betroffenen in die Datenbearbeitung möglich (S. 88, $106 \mathrm{ff}$.) und es sind auch keine sonstigen Rechtfertigungsgründe für die durch die drohnengestützte Datenbearbeitung entstehenden Persönlichkeitsverletzungen ersichtlich (S.108). Der Autor findet dafür klare Worte: Bei der drohnengestützten Daten-

1 Bundesverfassung der Schweizerischen Eidgenossenschaft vom 18. April 1999 (BV; SR101). bearbeitung werden regelmässig widerrechtliche Persönlichkeitsverletzungen begangen, wogegen den betroffenen Personen grundsätzlich Rechtsansprüche aus Art. 28 $\mathrm{ZGB}^{2}$ resp. Art. 32 nDSG ${ }^{3}$ zustehen würden. Diese können aber aufgrund der mangelnden Identifizierbarkeit der Drohnenbetreiber in der Regel gar nicht wahrgenommen werden. Ein effektiver Rechtsschutz fehlt somit (S.117).

Teil 4 widmet sich den datenschutzrelevanten Bestim- 8 mungen im Luftfahrtrecht, namentlich den neuen Drohnenverordnungen der EU, welche von der Schweiz aufgrund des Luftverkehrsabkommens Schweiz-EU4 zu übernehmen sind. Nach diesen Rechtsquellen müssen alle «Hobbydrohnen», die zur Datenbearbeitung in der Lage sind, registriert werden, und fallen nach Ansicht des Autors auch nicht unter die Ausnahme für «Spielzeugdrohnen» (S.125ff.). Aus datenschutzrechtlichen Gründen spricht sich der Autor für ein Drohnenbetreiberregister aus, das demjenigen des Registers für Strassenfahrzeuge in der Schweiz nachempfunden ist, das also nicht vollöffentlich ist, sondern nur auf Antrag der betroffenen Person (S.134). Zwar müssen Drohnen nach neuer EU-Rechtslage mit ihrer Betreibernummer eindeutig gekennzeichnet werden, allerdings ist zweifelhaft, ob die Betreibernummer im Flug tatsächlich gelesen werden kann. Damit können also nicht alle datenschutzrechtlichen Bedenken ausgeräumt werden (S.138). Von Bedeutung ist deshalb die ebenfalls neu eingeführte Möglichkeit zur Fernidentifizierung von Drohnen (Teil 2 Ziff.12, Teil 3 Ziff. 14 und Teil 4 Ziff. 9 Anhang der Delegierten Verordnung (EU)

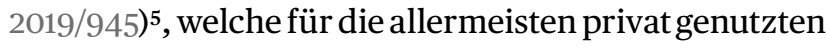
Drohnen zutreffen dürfte. Hierfür ist die Nutzung einer App oder einer Website notwendig. Der Autor sieht in der Fernidentifizierung, gemeinsam mit den Registrierungsvorschriften, ein grosses Potenzial, um die bisher fehlende Transparenz über die Drohnenbetreiber herzustellen (S. 142). Herausforderungen identifiziert er insbesondere bei der dafür notwendigen Mitwirkung der Drohnenbetreiber, welche allerdings durch in den Verordnungen vorgesehene flankierende Massnahmen wie Information und Aufklärung sowie eine Online-Theorieprüfung begegnet werden kann. Während der Autor diese Vorschriften als wesentlichen Schritt in die Richtung einer wirksamen Durchsetzung von Rechtsansprüchen gegenüber den Drohnenbetreibern ansieht (S. 155), weist er aber auch zu Recht darauf hin, dass in den Drohnenverordnungen

2 Schweizerisches Zivilgesetzbuch vom 10. Dezember 1907 (ZGB SR 210).

3 Entwurf vom 25. September 2020 zum Datenschutzgesetz (nDSG; $\mathrm{BBl} 20207639)$.

4 Abkommen zwischen der Schweizerischen Eidgenossenschaft und der Europäischen Gemeinschaft über den Luftverkehr vom 21. Juni 1999 (SR 0.748.127.192.68).

5 Delegierte Verordnung(EU) 2019/945 der Kommission vom 12. März 2019 über unbemannte Luftfahrzeugsysteme und Drittlandbetreiber unbemannter Luftfahrzeugsysteme. 
keine wirksame Möglichkeit zur Durchsetzung datenschutzrechtlicher Ansprüche vorgesehen ist: Registrierungs-, Kennzeichnungs- und Fernidentifizierungspflichten stellten zwar die Grundlagen dafür dar, doch fehle eine Verbindung mit dem Datenschutzrecht. Es bestehe somit immer noch eine Lücke im Rechtsschutz (S. 156).

Diese festgestellte Lücke im Rechtsschutz, die also sowohl im neuen Datenschutzgesetz wie auch in den (noch innerstaatlich umzusetzenden) EU-Drohnenverordnungen besteht, will der Autor in Teil 5 seiner Studie schliessen. Damit schliesst er den Kreis zu den in Teil 2 untersuchten Schutzpflichten: Es liege eine Situation vor, in welcher die grundrechtlich verankerten und im nDSG konkretisierten Ansprüche der betroffenen Person nicht mehr effektiv zur Geltung kommen. Die in Teil 2 identifizierten Voraussetzungen für das Entstehen einer Schutzpflicht seien deshalb erfüllt. Hierfür gehe es namentlich darum, zivilrechtliche Rechtsbehelfe zu ermöglichen, welche ausreichend Schutz bieten. Zusätzliche strafrechtliche Bestimmungen sind nach Ansicht des Autors nicht erforderlich (S. 161). Diese Einschätzung wird vom Autor nicht näher begründet, was bedauerlich erscheint (aber hier spricht womöglich bloss die passionierte Drohnengegnerin). Dass aber gesetzgeberische Handlungen notwendig sind, erscheint unzweifelhaft, und wird vom Autor auch damit begründet, dass ansonsten die Gefahr besteht, dass sich Private der Selbsthilfe oder Notwehr bedienen, und beispielsweise eine Drohne über ihrem Grundstück «abschiessen», was wiederum das staatliche Gewaltmonopol in Frage stellen würde (S.162).

10 Gestützt auf diese Überlegungen schlägt der Autor eine in das Luftfahrtgesetz aufzunehmende Bestimmung vor, welche dem Zweck der Durchsetzung von datenschutzrechtlichen Ansprüchen dienen soll. Namentlich plädiert er für die Schaffung eines neuen Art. 107c LFG ${ }^{6}$ (S. 165), welcher im Zuge der Umsetzung der Drohnenverordnungen in das LFG aufgenommen werden könnte. Der Autor legt hierzu einen kommentierten Formulierungsvorschlag vor, der vom Gesetzgeber bzw. der Verwaltung praktischerweise nur noch in den Entwurf und in die Botschaft kopiert werden müsste. Inhaltlich würde mit dem Formulierungsvorschlag ein schweizerisches «Drohnenbetreiberregister» geschaffen, welches alle Halter von privat genutzten Drohnen erfasst. Darin wären namentlich Namen und Geburtsdatum, Post- und E-Mail-Adressen, Telefonnummern sowie Versicherungspolicen der Drohnenbetreiber aufzuführen. Eine Datenbekanntgabe an Private wäre zwecks Geltendmachung von datenschutzrechtlichen Ansprüchen möglich, wofür nach Vorschlag des Autors ein Onlineformular des BAZL zur Verfügung

6 Bundesgesetz über die Luftfahrt vom 21. Dezember 1948 (Luftfahrtgesetz, LFG; SR 748.0). gestellt werden könnte. Zudem wäre ein (automatischer) Datenzugriff im Abrufverfahren für Strafverfolgungsund andere Behörden möglich. Der Vorschlag ist realistisch und erscheint geeignet, die vom Autor identifizierten Rechtsschutzlücken zu schliessen und somit für eine bessere Durchsetzung der datenschutzrechtlichen Betroffenenrechte zu sorgen.

\section{Fazit und Würdigung}

Dem sowohl formell wie inhaltlich überzeugenden Geset- 11 zesvorschlag sowie dem Buch insgesamt ist eine breite Leserschaft zu wünschen. Durch die open access Publikation sind hierzu beste Voraussetzungen vorhanden, und auch die angenehme Sprache von Henseler sowie die Kürze des Buches mögen dazu beitragen. Das Buch ist schnell gelesen, es ist konzis, zielgerichtet und informativ. Die Fragestellung ist präzis und praxisbezogen, was es dem Autor erlaubt, einen konkreten und gut begründeten Gesetzgebungsvorschlag zu formulieren, der vom Gesetzgeber in genau dieser Form im Zuge der nach wie vor laufenden Umsetzungsarbeiten der EU-Drohnenverordnungen übernommen werden könnte - was man dem Vorschlag denn auch sehr gönnen würde.

Die Kehrseite dieses Ansatzes ist ein für eine Promoti- 12 onsschrift doch recht geringer theoretischer Anspruch. Dass auf die strafrechtlichen Aspekte von drohnengestützten Aufnahmen im Privatbereich nicht eingegangen wird, ist bedauerlich. Noch grundlegendere Fragen, die durch die Thematik der drohnengestützten Datenbearbeitung aufgeworfen werden, tippt der Autor höchstens in Nebensätzen an, so etwa die zunehmende Aufweichung der Abgrenzung zwischen öffentlichem und privatem Raum (S. 4 f.) oder die allgemein bestehenden erheblichen Vollzugsdefizite im Datenschutzrecht (S. 157). Und wenn der Autor seine Studie mit den Worten einleitet, dass Drohnen «gewissermassen «den materiellen, auf die Welt zugreifenden Arm der Digitalisierung»» bilden und das Potenzial haben, «die iikonische Maschine» der Digitalisierung zu werden, wie dies einst die Dampfmaschine gewesen ist» (S.1), so würde man doch erwarten, zumindest im Schlusswort einige grundlegendere Überlegungen zur Rolle der Drohnentechnologie im Gesamtgefüge der gegenwärtigen digitalen Umwälzungen und deren Einflüssen auf das Recht (und umgekehrt) vorzufinden. So aber bildet diese Studie nur (aber immerhin) eine interessante Exemplifizierung der angesprochenen grundlegenden Problemfelder.

\section{Rezensiertes Werk:}

«Datenschutz bei drohnengestützter Datenbearbeitung durch Private», David Henseler, sui generis Verlag, Zürich 2020,

ISBN: 978-3-907297-10-0

(gedruckte Ausgabe, Hardcover; e-Book, Open Access) 
L'utilisation de drones par des particuliers necesse de croître et soulève d'importantes questions en matière de protection des données. Lespersonnes quientendentou voient un drone nesavent généralement passielles sonten train d'êtreobservées, si des enregistrements sont effectués et qui est responsable du drone. Une thèse de doctorat récemment publiée examine comment le droit à l'autodétermination en matière d'information peut être mis en ouvre lors du traitement de données récoltées par des particuliers à l'aide de drones. Létude analyse aussibien la nouvelle loi sur la protection des données que les dispositions relatives aux vols de drones et présente sur cette base des propositions concrètes d'adaptation de la loi. 